\title{
(Re-)Actions Speak Louder Than Words? A Novel Test Method for Tracking User Behavior in Web Video Services
}

\author{
Werner Robitza \\ T-Labs, Deutsche Telekom AG, Germany \\ Email: werner.robitza@telekom.de
}

\author{
Alexander Raake \\ Audiovisual Technology Group, TU Ilmenau, Germany \\ Email: alexander.raake@tu-ilmenau.de
}

\begin{abstract}
Assuring user engagement has become a key issue for Internet Service Providers and Over-the-Top Providers. How long are users consuming a service? When are they likely to abandon it due to quality problems? Rather than just estimating perceived audiovisual quality, future quality prediction models will also factor in possible user behavior. This contribution presents a novel test method to assess short-term user behavior in web video services, in a controlled living-room-like environment. We show that typical behavioral responses (such as seeking, reloading, or selecting another video) can be elicited, with the real purpose of the test hidden from the viewers. We can also see that when users are not focused on judging quality, their perception of errors changes significantly. This paper highlights the strong impact of laboratory test situations on users' behavior and discusses the challenges revolving around finding valid test methods.
\end{abstract}

Keywords-Quality of Experience; Behavior; Subjective Testing

\section{INTRODUCTION}

The continuous increase in Internet video traffic causes huge demands for the stakeholders involved in the delivery chain of Over-the-Top (OTT) services in terms of required peering and core network bandwidth. The experience of the customers at the end of this chain (Quality of Experience, QoE) is one of the main decision criteria - with a plethora of offered services, unhappy customers are assumed to be even more likely to churn and leave a provider for the one that delivers better experience [1].

Nowadays, quality models are the tool of choice for service providers to estimate the current level of experience for their customers. We can expect a perspective change in which providers will move from simple (post-fault) quality monitoring to a more individualized view on their customers, to deliver better quality and prevent users from churning. A key term here is engagement: A video provider may be more interested in raising the number of minutes their customers spend in a session (e.g., to increase advertisement exposure) or per-video rather than just minimizing the possible degradations that occur. We can therefore relate engagement to certain kinds of short-term behavior that we can observe on users: how long will a single video be watched before it is stopped? When do users quit a video session in the presence of problems? Current quality metrics generally do not consider these behavioral aspects. They may yield results in terms of mean opinion scores (MOS), but make no predictions on how users will interact with a system. This is also largely due to the fact that standardized subjective quality assessment methods do not allow for test participants to even behave like they would in real life. In this paper, we dive into the topic of human behavior and the associated challenges with user test methodologies. We present a new methodology for observing user behavior in the presence of video delivery problems and the results from a first study implementing it. It addresses many factors previously neglected in this kind of research. Section II will first present an overview of existing literature on this topic as well as motivate our studies. In Section III, we explain the theoretical background, then focus on video services in particular and list possible interaction patterns. Section IV describes our new test methodology and the study we conducted. Its results are shown in Section V. Section VI discusses the topics. Finally, We wrap up the paper in Section VII and give an outlook into our future work.

\section{BACKGROUND AND MOTIVATION}

Engagement is a key concept that has recently been studied more extensively with regard to OTT services. We can broadly see engagement as a special form of behavior in which users continue to use a service-mostly for certain affective reasons (e.g., feeling joy or not experiencing frustration) or because of more practical concerns (e.g., free vs. paid services), although the term has various concrete meanings in the literature, as we will see in the remainder of this section.

Already in 2005, Vilas et al. [2] analyzed user behavior on a video-on-demand ( $\mathrm{VoD}$ ) platform. Amongst other factors they looked at how often users paused or skipped videos. They developed a first conceptual state model of behavior in online video services. In [3], Dobrian et al. analyzed data from over two million video viewing sessions to study the impact of various video delivery characteristics on user engagement, defined as the video viewing time. Quality indicators (e.g., video load times) were related to engagement on different time scales: short-term (per video) and long-term (over multiple sessions). Balachandran et al. [4] used a dataset with 40 million views to extract rebuffering and loading time indicators. Based on those, they developed a predictive model of user engagement using decision trees. They proposed that engagement forms as a process of certain control parameters (e.g., in the network) influencing experienced quality, which in turn influences engagement. Their study therefore bridges between concrete network design choices and end user behavior. It also develops 
techniques to deal with confounding factors in such a largescale data analysis. A similar decision tree was shown in [5]: rather than predicting viewing time, the authors were interested in whether users complete or abandon video sessions. They directly related those outcomes to mobile network characteristics, rather than introducing a quality component as in [4]. In [6], a 23 million view dataset and the Quasi-Experimental Design (QED) method were used to infer statistical causality and significance between quality indicators (e.g., rebuffering duration) and viewer abandonment (aborted sessions), engagement (view time) and repeat viewership. Finally, in [7], a browser extension which tracks rebuffering events on YouTube is presented, with analysis relating rebuffering rates and quality switches to abandonment.

All those studies were performed on large-scale datasets, mostly identifying correlations and causal relationships between quality indicators and engagement as the target factor. Generally, they agree that longer loading times or higher rebuffering frequency lead to increased abandonment and that bandwidth and bitrate characteristics also play a large role in engagement prediction. Based on real customer viewing sessions, all above analyses however can only be performed on what happened in real networks. A structured experimental approach to identifying which event causes which kind of behavior will have to be followed in a laboratory environment, however, since there are many concerns associated with deliberately introducing degradations in real customer transmissions for experimental purposes [3]. Laboratory studies enable us to interact with test persons, ask them or measure how they feel, and-most importantly-specifically vary experimental factors so as to reduce the number of confounding variables in later analyses. Moreover, laboratory tests typically form the basis for developing QoE models applied for example in monitoring solutions within ISP networks. Here, respective laboratory tests can help to bridge the gap between engagement-related research and "standard" quality tests.

Mok et al. [8] investigated how certain network conditions relate to user behavior and interaction with video players in a laboratory environment. They listed different behavioral activities and described their implicit meanings (e.g., pausing may mean that users want to buffer more data). In their test, users were instructed to watch videos and told that they would experience different network performance levels. With a network traffic shaper, degradations in the form of video quality switches and rebuffering events were introduced. User behavior was logged in the video client, and users were asked to provide MOS ratings for every video transmission. The authors found that certain events like rebuffering led to user reactions like pausing the video or reducing the screen size. They also described the impact of different quality indicators and user behavior events on the MOS results.

While Mok et al. used network conditions as independent experimental factors, we believe it is necessary to go a step further and directly identify the relationship between perceptual events (such as long initial loading, rebuffering or quality drops) and user actions. Even more importantly, we want to elicit realistic and ecologically valid behavior: users should not be aware of the technical intricacies of the system under study-they should behave as naturally as possible without having the feeling of being watched, or even be asked for quality ratings while or after they interact with the system. We therefore developed a new, non-intrusive experimental methodology, further described in Section IV, based on a theoretical analyses of human behavior.

\section{ON BEHAVIOR AND INTERACTION}

What is behavior? For our purposes, we broadly define behavior as the response of a human to a stimulus. It can be overt (visible) or covert (invisible). Behavior does not necessarily have to relate to oneself; it can also be interactive. In that case we distinguish between human-to-human or human-to-machine interaction. Behavior can either be caused by unconscious (reflexive, intuitive) processes or conscious thought actions that require deliberation. Some forms of behavior (e.g., the show of joy or anger) are responses to certain internal affects (felt emotions). Affects have a positive or negative valence. Regarding time scale, typical behavioral actions happen within fractions of a second and last only few seconds at most. Long-term views on how people react over time are not part of the presented studies, and we do not call them "behavior".

What kind of behavior and interaction patterns are we expecting from users of online video services? Why do we expect them to behave in a certain way? Thorndike's Law of Effect [9] explains how humans repeatedly react the same way to certain conditions if they produce a satisfying effectthe opposite being true for negative experiences. When it comes to entertainment and media, we can assume that users generally want to maximize their level of positive experience and enjoyment. At the same time, they want to minimize their exposure to aversive events (i.e., stimuli that people want to avoid). Prolonged engagement with a stimulus-particularly meaning audiovisual entertainment in this case - can lead to a state of immersion, in which users "plunge" into the story, experiencing a kind of "flow" [10]. On the one hand, any interruption of that flow (e.g., through stalling events or bad quality) is thought to bring them back to a state of awareness of problems. It causes a reaction once the problem intensity reaches a certain threshold (e.g., a stalling event taking too long). On the other hand, users can maximize their immersion by taking different actions (e.g., turning on fullscreen mode). All this assumes that perceived quality and behavior (or action) are interrelated [11]. Indeed we can see how corrective behavior could change the quality perception-it is a process that naturally occurs all the time. Previous work that relates behavior to QoE on a systematic conceptual basis can be found in [12]. Reichl et al. present a framework that sees behavior as a consequence of a certain user state, which is in turn influenced by the system performance and QoE. They describe different effects of (system-, user- or context-related) influencing factors, either directly affecting QoE or behavior, or one through the other as a compound process. However, research work is still to be done to also address temporal factors and specify how quality, user states, and behavior interrelate.

For web video services, Table I lists typical (visible) interaction patterns, but it is not exhaustive. Notably, it only lists actions that are technically motivated, not motivated by the content. In other words, they are not related to how users want to watch contents (for example re-watching a segment because 
it was interesting, or stopping a video playback because the video was boring). We can primarily classify the (re)actions according to the valence of the stimulus that caused them. In other words: is this something people do because they have a great experience or something in response to problems? At first sight it may seem strange that there are fewer items in the positive valence domain. However, keeping in mind that entertainment services are designed to already deliver a high level of experience by default, there are few actions users may be able to take to even further increase their experienceespecially from a technical standpoint. It must be noted that different actions that result from technical problems may not be easily distinguishable from similar actions that are motivated by the content, the user's general preferences or external factors such as distractions and thus required interruptions or repetitions. The goal of the study presented in the next section is to elicit some of these patterns on the basis of problems due to a (simulated) slow network connection.

\section{HOW CAN BEHAVIOR BE ASSESSED NON-INTRUSIVELY?}

When we look at standardized and broadly used methods to assess audiovisual quality (e.g., ITU-T Rec. P.910), they all have many aspects in common. For example, users are seated in a laboratory room and are asked to passively listen to or view a number of stimuli. They are asked to select the preferred from two or more stimuli (preference rating) or provide quality ratings on predefined scales. These setups deny people two main possibilities that are essential to their real-life use of services: first, they cannot interact with the playout system using actions as outlined in Section III. Second-even though they may be exposed to degradations they may not tolerate in real-life - the only way to react to those is to give a bad quality rating in the end. To address the second aspect, one could imagine extending the existing methods to allow users to abort a sequence-but this carries additional consequences (e.g., missing ratings and the question of how such a reaction translates into MOS) and is beyond the scope of this paper. To tackle the first point, we describe a methodology that allows users to behave as they normally would. Note that at this stage of developing our methods, we are not interested in asking users for quality ratings.

\section{A. A novel test design}

While Mok et al. [8] already gave users the opportunity to interact with the video playback system, they did so by telling users about the purpose of the test and the different underlying network conditions. The study is also missing specific explanations as to which task was given to the users. We hypothesize that the awareness of the purpose of the study has major implications on how users behave in a test situation due to demand characteristics [13]. This effect describes that test participants may alter their behavior based on their interpretation of what the aim of a study is. For example, one could imagine that subjects trying to fill the "good-participant role" interact with a test system more than they do in real life, for example to give the experimenter data to use. Or, in the same role, they could endure extreme degradations because they do not want to admit any problems.
Our novel test method tries to counteract these effects and instead hides the real purpose of the experiment from the participants. We ask users to watch a number of sequences using a video portal with a layout similar to popular online video services. After they are greeted by the test supervisor, they are told that the purpose of the study is to assess how people consume online videos and how they describe video contents. They should imagine they were browsing one of their favorite online video portals. Then they are given a laptop that has a video portal open in a web browser. The portal presents them with a number of sequences that are representative of typical web-video content, and users may choose whatever sequence they like. After selecting a video from the overview screen, they can watch it entirely. When playback is finished, they are asked to summarize the content in a few sentences (as if they were recommending it to a friend), answer a contentrelated question (to prove that they have watched it) and rate how much they liked the clip. From all the clips that are seen, some of them include degradations typical of real video services, such as long rebuffering events or low quality periods. The interaction of users with the system is recorded unobtrusively in the background, unknown to the participant. The experimenter sits in another room but is available for questions. Only at the end of the test, the experimenter reveals the real purpose of the test: assessing behavior in response to quality degradations. What is the rationale behind these choices? The described tasks are intended to change the subjects' mindset while watching - away from spotting quality degradations. By letting people freely decide which video to watch, we expect to increase their intrinsic motivation. Through asking questions about the content itself, their extrinsic motivation to focus on the content instead of its quality is increased. At the same time, it prevents users from just skipping through every video, since they would not be able to answer content questions in this case. We followed the general approach described above to implement a first study, whose details will be described in the remainder of this section.

\section{B. Specific test implementation}

We selected 32 representative videos from existing web video portals. They belong to the categories animation, comedy, documentary, sports, and action. All videos were cut to a length of 1.5-3 minutes (if they were not already within that range) and showed a meaningful passage of the entire clip with appropriate starting and ending.

To run the test, we developed our own video portal (mimicking e.g., YouTube, albeit lacking all social features) using a web development framework. Users can access the portal through a standard web browser. The main page shows a grid of video thumbnails with their names and duration shown underneath. When the user selects a video, they are forwarded to a dedicated video page that implements a video player in the middle of the page (non-maximized). For this part, we used the player Clappr, keeping its entire functionality, but created a plugin that has the capability of adding "fake" stalling events, forced video quality switches, and simulating a buffer bar. To the users, it appears that the player is progressively downloading a video, with the buffer growing in chunks, while in fact the video is already fully cached on the test computer. When a stalling event occurs, users can seek backwards and resume playing immediately, but when seeking forward, they 
TABLE I. USER BEHAVIOR AND INTERACTION PATTERNS.

\begin{tabular}{|c|c|c|c|c|}
\hline Action & Target & Valence & (Possible) Intent & Comments \\
\hline Pause & Player & Negative & Pause playback for re-buffering & Typically requires player to indicate loading status \\
\hline Change to lower quality & Player & Negative & Choose matching bandwidth & Assumes that users know that quality relates to bandwidth \\
\hline Change to higher quality & Player & Positive & $\begin{array}{l}\text { Choose better quality, maximize quality for given band- } \\
\text { width }\end{array}$ & $\begin{array}{l}\text { See above. Is not necessary in case of good client adaptation algorithm, } \\
\text { but otherwise may help to overrule sub-optimal settings. }\end{array}$ \\
\hline Seek backward & Player & Negative & Re-play already loaded part, trigger re-loading & May not help in the case of slow network performance \\
\hline Seek forward & Player & Negative & Play future (unbuffered) portion to trigger re-buffering & See above \\
\hline Switch to fullscreen & Player & Positive & $\begin{array}{l}\text { Improve perception of details; reduce distraction due to } \\
\text { other website elements; increase immersion into media }\end{array}$ & \\
\hline Switch to windowed mode & Player & Negative & $\begin{array}{l}\text { Reduce resolution due to bad quality; prepare to interact } \\
\text { with page }\end{array}$ & \\
\hline Reload page & Page & Negative & Trigger full reload of video & May not help in the case of slow network performance \\
\hline Select other video & Session & Negative & Find a video that is working & $\begin{array}{l}\text { Assumes users relate specific video sequences with quality problems, } \\
\text { not just networking-related but e.g. due to (low) source quality }\end{array}$ \\
\hline
\end{tabular}

must wait for a predefined number of seconds ( $5 \mathrm{~s}$ in this case). When they switch the quality level, another waiting period is introduced - its length depending on whether they switch up $(5 \mathrm{~s})$ or down $(2 \mathrm{~s})$. It is also possible to enable fullscreen mode and change the player volume. To summarize, the system allows many of the basic interaction possibilities that are also found in real online portals-users are also free to go back to the main page and select another video or reload the browser tab.

We encoded all videos with ffmpeg's libx264 and aac as video and audio encoders, respectively, using five quality profiles. We selected profiles ranging from $420 \times 240$ pixels video resolution with $12 \mathrm{fps}$ framerate, $150 \mathrm{kBit} / \mathrm{s}$ video and $32 \mathrm{kBit} / \mathrm{s}$ audio bitrate at the lowest profile, up to $1920 \times 1080$ at $24 \mathrm{fps}$ with $4.5 \mathrm{MBit} / \mathrm{s}$ video and $128 \mathrm{kBit} / \mathrm{s}$ audio bitrate at the highest profile. The quality levels thus spanned a range from noticeably bad to excellent audiovisual quality. Videos were segmented into 2-second MPEG-TS chunks, from which we created HTTP Live Streaming (HLS) playlists that were then used by the playback software. The profiles were chosen in such a way that a quality switch between them should be noticeable. Users then saw the following conditions:

1) Reference: Video shown with highest quality, no initial loading, stalling or quality changes

2) Initial loading: $30 \mathrm{~s}$, which according to previous literature (see Section II) was expected to not be tolerated by most users.

3) Long stalling: $30 \mathrm{~s}$ inserted at 00:30, same motivation as with 2)

4) Quality drop: From highest to lowest level at 00:45

5) Medium quality: Video shown at quality level 3, no initial loading, stalling or quality changes

6) Low quality: Same as 5), but video at quality level 1 (lowest)

The conditions were assigned to the videos in the following manner: The first video that subjects selected always showed the reference condition. For every subsequent video played, another condition was randomly picked from the entire set of conditions minus the reference (drawing without replacement). At the end, the reference was shown again. This means that users saw the reference twice and every other condition once, totaling in seven videos. With an average of 2-3 minutes, this resulted in a watching time of about 25 minutes (including answering the content questions).
The test was conducted in a living room-like environment, with leather furniture, a coffee table, a big TV screen in the background, carpets etc. To watch the videos, the participants were given a 13" Retina MacBookPro $(2880 \times 1800$ native resolution). This allowed them to view the video in full HD if wanted. We explicitly allowed them to hold it however they liked and adjust the volume to their preference.

\section{RESUlts}

We ran the test described above with 15 participants (aged 24-60, median 29, 8 female). Participants were mostly students (9), and all of them had completed a traditional audio or video quality test before. The majority watched online videos at least once a day. We now give an overview of the results, with a focus on a qualitative description of the user behavior.

\section{A. Observed behavior and interaction patterns}

Before addressing specific reactions to stalling/loading events or quality problems, we categorize the types of test participants, depending on whether they reacted to those problems at all, according to three criteria:

- Non-reacting vs. Reacting: Whether participants reacted to quality degradations or stalling events at all, or whether they just waited and did nothing.

- Apprehensive vs. Unaware: Apprehensive subjects did not react (or changed their reactions) because they were influenced by the test situation (see below), as evaluated through interviews with the participants.

- Calling vs. Non-Calling: Users who called the experimenter at some point because of problems.

We see "apprehensive participants" as the most problematic artifact of the study results or of this kind of test procedure as a whole. The concept is related to the "good participant" hypothesis [14], which describes subjects who think they understand the hypothesis behind an experiment and will show behavior that proves this hypothesis. While the "real" hypothesis to prove in this study would have been that people indeed show reactions-which the majority did-, our "good participants" did not want to tamper with the test software (for example by reloading the page), despite being told to watch as they would in real life. Table II marks participants who, according to their own description, were acting apprehensively. Participants acting in such a way distort the results of a study, as they may have showed different reactions in a typical watching situation 
TABLE II. OVERVIEW OF REACTIONS TO CONDITIONS. ( $F=$ Fullscreen, $R=$ reload page, $P=$ pause, $S=$ seek, $V=$ select other video $)$

\begin{tabular}{|c|c|c|c|c|c|c|c|c|c|c|c|c|c|c|c|c|}
\hline Subject & S1 & S2 & S3 & S4 & S5 & S6 & S7 & S8 & S9 & S10 & S11 & S12 & S13 & S14 & S15 & Percent \\
\hline Apprehens.? & $x$ & $x$ & $x$ & $\checkmark$ & $x$ & $x$ & $\checkmark$ & $x$ & $\checkmark$ & $x$ & $\checkmark$ & $x$ & $x$ & $x$ & $x$ & $27 \%$ \\
\hline Calling? & $x$ & $x$ & $x$ & $x$ & $\checkmark$ & $x$ & $\checkmark$ & $x$ & $\checkmark$ & $x$ & $x$ & $x$ & $x$ & $\checkmark$ & $x$ & $27 \%$ \\
\hline Ref 1 & - & - & FF & $\mathrm{F}$ & - & - & - & F & F & - & - & - & - & - & - & $27 \%$ \\
\hline $\operatorname{Ref} 2$ & - & - & $\mathrm{F}$ & - & - & - & - & - & $\mathrm{F}$ & - & - & - & - & - & - & $13 \%$ \\
\hline Initial load & - & - & FFSVS & - & RSVS & - & RVSFF & $\mathrm{P}$ & $\mathrm{F}$ & $3 \times S$ & - & - & RVVRRSSVVRSS & $\mathrm{RR}$ & - & $53 \%$ \\
\hline Long stall & - & - & FSVFFSF & - & $\mathrm{S}$ & - & FFVS & - & $\mathrm{F}$ & $5 \times \mathrm{S}$ & - & $\mathrm{S}$ & SS & - & - & $47 \%$ \\
\hline Quality Drop & - & - & $\mathrm{F}$ & - & - & - & - & - & $\mathrm{F}$ & - & - & - & - & - & - & $13 \%$ \\
\hline Med. Quality & - & - & $\mathrm{F}$ & - & - & - & - & - & $\mathrm{F}$ & $\mathrm{P}$ & - & - & - & - & - & $20 \%$ \\
\hline Low Quality & - & - & $\mathrm{F}$ & - & - & - & $\mathrm{FF}$ & - & - & - & - & - & - & - & - & $13 \%$ \\
\hline
\end{tabular}

at home. We identify this as a key issue to address in this kind of research. It should be noted that unexpectedly, Subject 7 described herself as apprehensive while still showing reactions. The reaction may be explained by the experimenter's presence after the subject calling him.

1) Loading/Stalling events: All of the participants noticed stalling degradations (conditions 2 and 3). Especially when the experimenter asked them, "Did everything work well?", every subject mentioned that some videos showed long loading periods, but some of the participants cared less about the problems than others. Those who showed any reactions at all did so only in response to the stalling events. This finding is not surprising: such long stalling events are typically associated with user abandonment or very low quality ratings. Looking at Table II, which shows the users' individual reactions, we can see that long initial loading mostly prompted users to a) select another video, b) reload the page and c) seek a bit (which caused the player to resume after 5 seconds). For the long stalling event, no page reloads were triggered. This could be explained by users wanting to continue watching a video once they had started it. There was a small tendency for more activity in case of initial loading than in case of stalling. This may be a side effect of the test task: subjects may have found initial loading to be more detrimental to the task of video summarization than stalling, where they already had collected some information for their later answer. Subject 7 and 13 showed a strong reaction where they repeatedly selected other videos or reloaded pages, more than once.

2) Low quality or quality drops: To our surprise, low quality playout of the video (in conditions 4,5 , and 6) was never causing reactions, no matter if it was shown throughout the video or in sudden drops. In contrast, many studies associate low quality ratings with strong quality drops [15]. While we did not ask for MOS ratings, we expected some kind of reaction by the users. When asking participants whether they noticed any low quality or quality drops, they indeed agreed, and the absence of reaction was explained differently depending on the participant. We collected the following explanations: a) users did not perceive any quality issues at all, b) users did not care because the video was so entertaining, c) users did not think or know they could change the quality, and d) users thought the video was uploaded in bad quality. We find these explanations very interesting because they highlight the importance of the task and viewing context: when users are consuming video for entertainment purposes, focusing on the content rather than rating the (video) quality, they may not even be aware of coding- or representation-related artifacts. This confirms results from the study in [16]Also, the (lack of) reaction shows that users may not be able to discern quality switches due to network problems from source video quality issues.

\section{DISCUSSION}

Is the proposed method suitable for eliciting the expected behavioral responses to quality degradations? There is no clear answer yet: we indeed observed most of the interaction patterns described in Section III, but not from all participants. Many did not interact with the system, either because they were trying to be a "good participant" or because they were more tolerant to errors in the present test situation. Here, the chosen task may have played an important role as well. When asked for quality in more traditional tests, subjects are thought to have a different focus on certain effects than in the case of watching videos for their own entertainment (as shown in [16]), often being more critical. In turn, in case of our summarization / liking task, the subjects' behavior is likely to be a mix between a more ecologically valid free watching of (in our case) rather entertaining contents and the corresponding behavior, combined with an approach to optimize the own task performance and hence to suppress possibly detrimental interactions with the setup. To investigate whether the test environment is the most influencing factor, such a test should ideally be conducted directly on real web video portals. This would however introduce dependencies on the ISP and video service provider, potentially resulting in unforeseen streaming conditions.

However realistic the video portal may be, we can see from the results that the behavior of participants is still very strongly influenced by their previous experiences and awareness of the test situation. Particularly in our sample, users with previous "video quality testing" experience were very reluctant to do anything against degradations they described as intolerable in real life - they would rather wait it out. Also, there was a hint in the instructions telling participants to watch videos entirely, which some may have interpreted as "in one go", discouraging them from selecting another video in the case of problems. As a consequence, it seems imperative to recruit nave subjects for behavioral tests. Even more importantly, the test protocol should make it clear that any kind of interaction is allowed, without revealing that those actions would be monitored. More generally, the findings from this study stress the non-negligible impact that different test protocols may have, which calls for even more ecologically valid test methods. How these can be achieved-by changing the context to the users' homes, improving the test software, etc.-is still an open discussion point. 
How can results from tests such as the one described in this contribution be exploited? As explained in the beginning, there is a strong interest to predict user engagement from certain quality indicators, and also assess how behavior influences the perceived quality of a video. This requires a well-designed test series that varies different degradation conditions, to allow models that, for example, predict categorical outcomes (user aborting playback, skipping forward, etc.) from certain events (long stalling, quality drops, etc.). On the one hand, the number of conditions that could be tested in this study was intentionally kept very small: inserting even more problems would give away the purpose of the test and could cause participants to behave differently. On the other hand, the results from a test with only few conditions cannot be used for the development of quality or behavior prediction models - unless a large number of studies are conducted. A combination of large-scale analyses (e.g., as shown in [3], [6]) with dedicated laboratory tests may however yield much more insight into why people behave in a certain way and help us qualitatively understand their behavior. Conditions and the behavioral responses seen in reallife could then also be replicated in the lab. Collecting quality ratings after video sessions in real services would also provide important feedback for such kind of research.

\section{CONCLUSION AND FUTURE WORK}

In this paper, we presented the topic of behavioral assessment in web video services as an essential tool to describe the underlying principles of user engagement and quality perception, with a strong tie to how customers use services in real-life. After showing related work on large-scale user engagement studies, we gave an introduction into the theoretical foundations of behavior and interaction. We then presented a new test methodology that elicits short-term reactions to quality problems that typically occur in web video services due to low network performance. The results show that some subjects did not react as they may likely have in real life, due to either being primed from having completed other video quality tests before, or trying to be a "good participant". For the remaining panel, we saw various typical interaction patterns in response to the degradations we inserted, except for coding quality degradations, which have mostly gone unnoticed or were ignored, partly as an artifact of the test, but mostly because users did not care.

In future studies, we not only want to examine the data from this study in more depth-for example, by inspecting reaction times to certain events and semantics of content summaries. We also want to address four points: 1) Increasing the (ecological) validity of the test procedure itself, making it even less intrusive. This could be achieved by more close-tolife test software and a more liberal protocol. 2) Extending the behavioral assessment to involuntary reactions by using eyetrackers or methods from physiological QoE research (e.g., brain activity [17]). This would give insight into internal affects, but at the same time make the test procedure less natural. 3) Expanding the studies to users' homes and longer time spans, which also should address the first point. 4) Including quality ratings to assess the relation between behavior and quality perception (which also requires identifying how much the task of having to provide a quality rating influences behavior).
Behavioral studies are still relatively rare in the QoE domain. Many questions remain to be answered, and they strongly relate to how we should even perform such tests: How do quality and behavior interrelate? How can test methods even be designed so that they capture behavior in an ecologically valid way? How does short-term behavior influence customer engagement?

\section{ACKNOWLEDGMENTS}

This work was funded from the European Unions Horizon 2020 research and innovation programme under the Marie SklodowskaCurie grant agreement No 643072, Network QoE-Net.

\section{REFERENCES}

[1] Conviva, "2015 Viewer Experience Report," Conviva, Tech. Rep., 2015.

[2] M. Vilas, X. G. Pañeda, R. García, D. Melendi, and V. G. García, "User behaviour analysis of a video-on-demand service with a wide variety of subjects and lengths," in Proc. Software Engineering and Advanced Applications, 2005, pp. 330-337.

[3] F. Dobrian, A. Awan, D. Joseph, and A. Ganjam, "Understanding the impact of video quality on user engagement," Communications of the $A C M$, vol. 56, no. 3, pp. 91-99, 2013.

[4] A. Balachandran, V. Sekar, A. Akella, S. Seshan, I. Stoica, and H. Zhang, "Developing a Predictive Model of Quality of Experience for Internet Video Categories and Subject Descriptors," ACM SIGCOMM Computer Communication Review, vol. 43, no. 4, pp. 339-350, 2013.

[5] M. Zubair Shafiq, J. Erman, L. Ji, A. X. Liu, J. Pang, and J. Wang, "Understanding the Impact of Network Dynamics on Mobile Video User Engagement," in Proc. SIGMETRICS. New York, USA: ACM, 2014, pp. 367-379.

[6] S. S. Krishnan and R. K. Sitaraman, "Video stream quality impacts viewer behavior: Inferring causality using quasi-experimental designs," IEEE/ACM Transactions on Networking, vol. 21, no. 6, pp. 2001-2014, 2013.

[7] H. Nam, K.-H. Kim, and H. Schulzrinne, "QoE Matters More Than QoS: Why People Stop Watching Cat Videos," in Proc. IEEE INFOCOM, 2016

[8] R. K. P. Mok, E. W. W. Chan, X. Luo, and R. K. C. Chang, "Inferring the QoE of HTTP video streaming from user-viewing activities," in Proc. ACM SIGCOMM W-MUST, 2011, p. 31.

[9] R. J. Herrnstein, "On the law of effect," Journal of the experimental analysis of behavior, vol. 13, no. 2, pp. 243-266, 1970.

[10] M. Csikszentmihalyi, "Flow and the psychology of discovery and invention," New Yprk: Harper Collins, 1996.

[11] A. Raake and S. Egger, Quality of Experience. Advanced Concepts, Applications and Methods. Berlin-Heidelberg-New York NY: Springer, 2014, ch. Quality and Quality of Experience.

[12] P. Reichl, S. Egger, S. Möller, K. Kilkki, M. Fiedler, T. Hossfeld, C. Tsiaras, and A. Asrese, "Towards a Comprehensive Framework for QoE and User Behavior Modelling," in QoMEX, 2015, pp. 0-5.

[13] M. T. Orne, "Demand characteristics and the concept of quasi-controls," in Artifacts in Behavioral Research: Robert Rosenthal and Ralph L. Rosnow's Classic Books, 2009, p. 110.

[14] A. L. Nichols and J. K. Maner, "The good-subject effect: Investigating participant demand characteristics," The Journal of general psychology, vol. 135, no. 2, pp. 151-166, 2008.

[15] M. N. Garcia, F. De Simone, S. Tavakoli, N. Staelens, S. Egger, K. Brunnström, and A. Raake, "Quality of Experience and HTTP Adaptive Streaming: A Review of Subjective Studies," in QoMEX, 2014, pp. 141-146.

[16] N. Staelens, S. Moens, W. Van den Broeck, I. Marien, B. Vermeulen, P. Lambert, R. Van de Walle, and P. Demeester, "Assessing quality of experience of IPTV and video on demand services in real-life environments," IEEE Transactions on Broadcasting, vol. 56, no. 4, pp. 458-466, 2010.

[17] J.-N. Antons, S. Arndt, R. Schleicher, and S. Möller, "Brain activity correlates of quality of experience," in Quality of Experience. Springer, 2014 , pp. $109-119$. 